\title{
DIVERSIDAD MORFOLÓGICA MANDIBULAR DE LOS MONOS CAPUCHINOS: UN ANÁLISIS MORFOMÉTRICO
}

\section{MORPHOLOGICAL DIVERSITY OF THE MANDIBLE OF CAPUCHIN MONKEYS: A MORPHOMETRIC ANALYSIS}

\author{
Joaquin del Rio', Sergio Furtado dos Reis², Thaís Maria Pires dos Santos³, Ricardo T. Lopes ${ }^{3}$ y S. \\ Ivan Perez ${ }^{4}$ \\ ${ }^{1}$ Facultad de Ciencias Naturales y Museo. Universidad Nacional de La Plata. La Plata. Argentina \\ ${ }^{2}$ Departamento de Biologia Animal. Universidade Estadual de Campinas. São Paulo. Brazil \\ ${ }^{3}$ Laboratório de Instrumentação Nuclear. Centro de Tecnologia. Universidade Federal do Rio de Janeiro. Rio de Janeiro. Brazil \\ ${ }^{4}$ CONICET. División Antropología. Facultad de Ciencias Naturales y Museo. Universidad Nacional de La Plata. La Plata. Argentina
}

PALABRAS CLAVE morfometría geométrica; Sudamérica; Cebus-Sapajus; sistemática

\begin{abstract}
RESUMEN La diversificación de los platirrinos a escala macroevolutiva ha sido sistemáticamente estudiada en años recientes. Sin embargo, el origen de la diversidad fenotípica y de taxones observada a escalas filogenéticas menores, p. ej., dentro de los géneros, ha sido menos estudiada. En este contexto, la diversidad de los monos capuchinos ha sido discutida recientemente. Los trabajos previos reconocían un único género y principalmente cuatro especies. En cambio, algunos estudios recientes, empleando datos morfológicos y moleculares, han propuesto dividir el clado en dos géneros (Cebus y Sapajus) y doce especies. El objetivo de este trabajo es explorar la variación morfométrica mandibular de las especies de monos capuchinos para discutir este problema. Los resultados obtenidos -empleando microtomografías computadas, técnicas de la mor-
\end{abstract}

fometría geométrica en 3D y métodos comparativos filogenéticos- muestran que todas las especies estudiadas se encuentran superpuestas a lo largo del eje principal de variación en forma, y que la diferenciación de las especies que recientemente han sido asignadas a los géneros Cebus y Sapajus se relaciona con cambios no alométricos en forma. Muchas de las características asignadas al recientemente propuesto género Sapajus, incluyendo particularmente la robustez de la mandíbula, son observadas en individuos masculinos de especies de ambos géneros. Estos resultados sugieren que las características morfológicas descritas recientemente para diferenciar los géneros de capuchinos deben ser empleadas con cuidado como criterios para generar nuevas propuestas sistemáticas. Rev Arg Antrop Biol 20(2), 2018. doi:10.17139/raab.2018.0020.02.07

\section{KEY WORDS geometric morphometrics; South America; Cebus-Sapajus; systematics}

ABSTRACT The diversification of platyrrhines on a macroevolutionary scale has been systematically studied in recent years. However, the origin of the phenotypic and taxonomic diversity observed at small phylogenetic scales, e.g., within genera, has been less studied. In this context, the diversity of capuchin monkeys has recently been discussed. Previous works recognized a single genus and mainly four species. In contrast, recent studies, using morphological and molecular data, have proposed splitting the clade into two genera (Cebus and Sapajus) and twelve species. The objective of this work is to explore mandibular morphometric variation of capuchin monkey species to discuss this problem. The results obtained-using computed micro-tomogra-

La diversidad de un clado es producto de las tasas de nacimiento y muerte de las especies, así como de los procesos aleatorios y determinísticos que actúan sobre el fenotipo durante la divergencia evolutiva del mismo (Schluter, 2000; Losos y Mahler, 2010). La diversificación de los platirrinos a escala macroevolutiva ha sido sistemáticamente estudiada en años recientes. Los resultados obtenidos sugieren que los platirrinos se diversificaron en las características morfológicas que diferencian los clados principales, i.e., phies, 3D geometric morphometric techniques, and comparative phylogenetic methods-show that all the species studied overlap along the main axis of shape variation, and that the differentiation among the species recently assigned to the genera Cebus and Sapajus is related to non-allometric shape changes. Many of the characteristics assigned to the recently proposed Sapajus genus, particularly, the robustness of the mandible, are observed in male individuals of species of both genera. These results suggest that the morphological characteristics recently described as useful for differentiating the capuchin genera should be cautiously used as criteria for generating new systematic proposals. Rev Arg Antrop Biol 20(2), 2018. doi:10.17139/raab.2018.0020.02.07

Financiamiento: Universidad Nacional de La Plata PI-787. Fondo para la Investigación Científica y Tecnológica PICT1810. Consejo Nacional de Investigaciones Científicas y Tecnológicas PIP-0603. Conselho Nacional de Desenvolvimento Científico e Tecnológico y Fundação de Amparo à Pesquisa do Estado de São Paulo.

*Correspondencia a: S. Ivan Perez. División Antropología, Facultad de Ciencias Naturales y Museo. 122 y 60. La Plata. E-mail: ivanperezmorea@gmail.com

Recibido 24 Julio 2017; aceptado 7 Enero 2018

doi:10.17139/raab.2018.0020.02.07 
subfamilias y géneros, en momentos tempranos de su historia de divergencia filogenética a lo largo de un nicho ecológico multidimensional, donde la dieta y el tamaño del grupo social fueron dos dimensiones principales (Arístide, dos Reis, Machado, Lima, Lopes, y Perez., 2015, 2016; Marroig y Cheverud, 2001; Rosenberger, 1992). Sin embargo, el origen de la diversidad fenotípica y de taxones observada a escalas filogenéticas menores, e.g., dentro de los géneros, no es completamente comprendido y ha sido fuertemente discutido en años recientes.

Entre los casos más discutidos se pueden destacar a los monos del género Cebus, comúnmente conocidos como monos capuchinos, que se encuentran ampliamente distribuidas en toda América Central y del Sur (Arístide, Soto, Mudry y Nieves, 2014; Fleagle, 2013; Lynch Alfaro et al., 2012a). Los trabajos efectuados hasta 2010 reconocían principalmente un único género y cuatro especies: Cebus capucinus, Cebus olivaceus, Cebus albifrons y Cebus apella. Esta última especie fue tratada como indiferenciada y ampliamente distribuida en Sudamérica (Wright et al., 2015). Sin embargo, algunos estudios efectuados en los últimos años han propuesto dividir el clado en dos géneros y doce especies (surgidas principalmente de la división de Cebus apella): Cebus capucinus, $\mathrm{Ce}$ bus olivaceus, Cebus kaapori, Cebus albifrons, Sapajus apella, Sapajus libidinosus, Sapajus flavius, Sapajus xanthosternos, Sapajus macrocephalus, Sapajus robustus, Sapajus nigritus y Sapajus cay (Lynch Alfaro et al., 2012a; Wright et al., 2015). El nuevo género Cebus se distribuye principalmente al norte de la cuenca amazónica y Centro América, mientas que el género Sapajus se encuentra en la mata atlántica, con una amplia área de simpatría en la cuenca del Amazonas (Lynch Alfaro et al., 2012a; Wright et al., 2015).

Los trabajos que plantearon la división del clado en dos géneros emplearon como evidencia a favor de su propuesta la divergencia crono-filogenética entre las especies del mismo -estimada con datos moleculares en ca. 6 millones de años atrás-, la distribución geográfica de las mismas y, principalmente, los patrones de diferenciación morfológica. En primer lugar, se mostró que el inicio de la diversificación de las especies de cada nuevo género comenzó en los últimos 2 a 2,5 millones de años, sugiriendo la evolución independiente de los mismos por ca. 4 millones de años en la mata Atlántica y la cuenca del Amazonas (Lynch Alfaro et al., 2012a). En segundo lugar, los trabajos han mantenido que los dos géneros se corresponden con especies morfológicamente robustas (género Sapajus) y gráciles (género Cebus). En particular, se empleó evidencia morfológica cualitativa y morfométrica tradicional (i.e., basada en distancias lineales entre puntos anatómicos; Adams et al., 2004) para señalar la diferenciación en la robustez morfológica craneal y mandibular entre las especies de Cebus de la cuenca amazónica y aquéllas de Sapajus de la mata Atlántica (Lynch Alfaro, Silva Jr., y Rylands, 2012b; Wright et al., 2015).

Sin embargo, hasta el presente se efectuaron escasos estudios sistemáticos empleando técnicas que permitan explorar de manera detallada la variación morfológica entre las especies asignadas recientemente a los géneros Cebus y Sapajus (e.g., Nova Delgado, Galbany, Górka, y Pérez-Pérez, 2015). Particularmente, el objetivo de este trabajo es estudiar la variación morfométrica mandibular de las especies asignadas a Cebus y Sapajus empleando una aproximación que permita describir detalles de las diferencias en forma entre las mismas. Con este fin se emplean micro-tomografías computadas, técnicas de la morfometría geométrica en 3D y métodos comparativos filogenéticos que permiten una descripción detallada de la variación en forma y tamaño en un contexto filogenético (Felsenstein, 1985; Blomberg et al., 2003; Mitteroecker y Gunz, 2009). En particular, debido a que trabajos previos han sugerido que las especies de Cebus y Sapajus se diferencian en tamaño y robusticidad, en este trabajo exploramos la variación en forma, tamaño, forma alométrica y no-alométrica empleando tanto landmarks como landmarks más semilandmarks (Drake y Klingenberg, 2008; Mitteroecker y Gunz, 2009). Asimismo, investigamos la estructura filogenética de las variables morfométricas estudiadas (Blomberg, Garland Jr. e Ives, 2003; Felsenstein, 1985). Los resultados obtenidos aportarán a la discusión sobre los procesos responsables de la diversificación del clado de los monos capuchinos, así como los criterios empleados en nuevas propuestas sistemáticas. 


\section{MATERIAL Y MÉTODOS}

Se analizaron un total de 55 tomografías computadas (CT) y micro-tomografías computadas $(\mu \mathrm{CT})$ de especímenes pertenecientes a 8 especies de los géneros Cebus (N=2) y Sapajus ( $\mathrm{N}=6$; Primates, Platyrrhini), distribuidos en Sudamérica y Centroamérica. Las especies muestreadas representan el rango total de variación geográfica y filogenética del clado, por lo que el muestreo es adecuado para discutir la variación entre los géneros. La especie a la que pertenece cada espécimen se determinó usando la información disponible en la colección de pertenencia, junto con la información geográfica del sitio de recolección de los mismos y mapas de distribución disponibles en la Red List de la IUCN (Rylands, Mittermeier y Silva, 2012). Se analizaron sólo individuos adultos, determinados como tales aquellos con una dentición totalmente erupcionada. Asimismo, se incluyeron especímenes de ambos sexos en similar proporción en todas las especies estudiadas (Tabla 1). Las CTs y $\mu$ CTs obtenidas pertenecen a especímenes depositados en los siguientes museos: Museu Nacional, Rio de Janeiro (Brasil; MNRJ), Museu de Zoologia da Universidade de São Paulo, São Pablo (Brasil; MZUSP), Museo de La Plata, La Plata (Argentina; MLP), Museo Argentino de Ciencias Naturales, Buenos Aires (Argentina; MACN) National Museum of Natural History, Smithsonian Institution, Washington (Estados Unidos; USNMDC) y Kyoto

TABLA 1. Composición de la muestra de Cebus-Sapajus clasificada según especie y sexo

\begin{tabular}{lccc}
\hline Especie & Hembras & Machos & Total \\
\hline C. capucinus & 3 & 5 & 8 \\
C. albifrons & 6 & 6 & 12 \\
C. (S) apella & 4 & 4 & 8 \\
C. (S) libidinosus & 3 & 3 & 6 \\
C. (S) nigritus & 3 & 3 & 6 \\
C. (S) macrocephalus & 2 & 3 & 5 \\
C. (S) cay & 4 & 1 & 5 \\
C. $($ S) robustus & 2 & 3 & 5 \\
\hline Total & 27 & 28 & 55 \\
\hline
\end{tabular}

University Primate Research Institute, Kyoto (Japan; KUPRI).

Las CTs y $\mu$ CTs fueron procesadas en el programa 3D Slicer 4.6 (www.slicer.org) para obtener superficies o imágenes en $3 \mathrm{D}$, que fueron guardadas en formato PLY. Sobre estas imágenes 3D se digitalizaron un total de 16 landmarks y 198 semilandmarks de curva (Fig. 1) usando el programa Landmark (Wiley et al., 2005). Los landmarks fueron ubicados en la región dental, mentón, extremos de apófisis, gonion y en la articulación mandibular, que son zonas de relevancia anatómica y fácilmente homologables entre individuos (Tabla 2). Con el fin de describir la forma de la mandíbula de la manera más completa posible, los semilandmarks de curva se ubicaron en las crestas temporales, mentón,
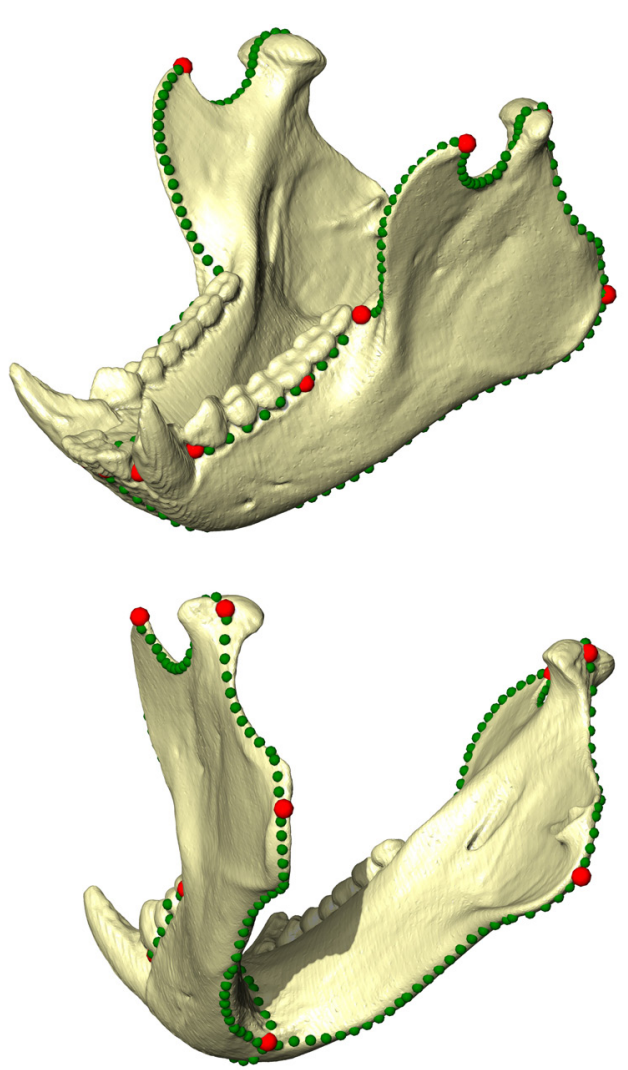

Fig. 1. Landmarks (en rojo) y semilandmarks (en verde) registrados sobre las mandíbulas de monos capuchinos. El punto localizado en el gonion fue considerado como semilandmark cuando se analizaron todos los puntos de referencia (i.e., landmarks y semilandmarks) de manera conjunta. 
TABLA 2. Definición de los landmarks y semilandmarks utilizados

\begin{tabular}{|c|c|}
\hline Puntos de referencia & Descripción \\
\hline \multicolumn{2}{|l|}{ Landmark } \\
\hline Condylion laterale (cdl) & El punto más posterior del cóndilo mandibular \\
\hline Infradentale (id) & Punto medio entre los incisivos 1 \\
\hline Canino-incisivo (ci) & Punto entre los caninos y el incisivo 2 \\
\hline Final dientes (fd) & Extremo posterior de la serie yugal sobre el borde labial \\
\hline Coronoide (crd) & Extremo de la apófisis coronoide \\
\hline Extremo-mentón (emn) & El extremo anterior e inferior del mentón \\
\hline Canino-premolar (cm) & Punto entre el los caninos y el premolar 1 \\
\hline Ectomolare (ect) & Punto medio labial del molar 1 \\
\hline Gonion (gn) & Punto sobre la curvatura máxima del gonion \\
\hline \multicolumn{2}{|l|}{ Semilandmark } \\
\hline Mentón & $\begin{array}{l}\text { Línea media desde el punto entre los incesivos } 1 \text { hasta el extremo anterior } \\
\text { e inferior del mentón, desde el landmark id hasta el emn. } 10 \text { puntos }\end{array}$ \\
\hline Gonion & $\begin{array}{l}\text { Borde posterior de la rama mandibular y borde inferior del cuerpo, desde } \\
\text { el landmark cdl hasta el fnp. } 39 \text { puntos en cada lado }\end{array}$ \\
\hline Serie yugal & $\begin{array}{l}\text { Sobre el borde labial de los dientes, desde el extremo posterior del canino } \\
\text { hasta el antrior del molar } 3.10 \text { puntos en cada lado }\end{array}$ \\
\hline Cresta temporal anterior & $\begin{array}{l}\text { Borde anterior de la cresta temporal, desde fd hasta crd. } 20 \text { puntos en cada } \\
\text { lado }\end{array}$ \\
\hline Cresta temporal posterior & $\begin{array}{l}\text { Borde posterior de la cresta temporal, continuando hasta el extremo más } \\
\text { posterior del cóndilo mandibular, desde crd hasta cdl. } 20 \text { puntos en cada } \\
\text { lado }\end{array}$ \\
\hline Fosa sublingual & $\begin{array}{l}\text { Linea media de la fosa sublingual, desde el punto medio entre los incisivos } \\
1 \text { hasta extremo del mentón (emn). } 10 \text { puntos }\end{array}$ \\
\hline
\end{tabular}

región dental yugal y sobre la curva inferior externa de la mandíbula (Fig. 1; Tabla 2).

Las coordenadas cartesianas de landmarks y semilandmarks fueron superpuestas empleando un Análisis Procustes Generalizado (Rolhf, 1990; Mitteroecker y Gunz, 2009). Este método permite eliminar la variación en posición, escala y orientación de los casos comparados, obteniéndose variables de forma denominadas coordenadas de forma Procrustes. Los semilandmarks de curva fueron deslizados para eliminar la variación tangente a las mismas empleando el criterio de Bending energy (Mitteroecker y Gunz, 2009). La superposición Procrustes, el deslizamiento de los semilandmarks y la obtención de las coordenadas Procrustes fueron realizadas en el paquete geomorph (Adams y
Otarola-Castillo, 2013) del programa R (R Development Core Team, 2017). Las coordenadas de forma Procrustes de los landmarks fueron analizadas de manera aislada y en conjunto con las coordenadas de los semilandmarks.

La asociación entre el tamaño y la forma mandibular fue explorada empleando un modelo de Regresión Multivariada, donde las coordenadas de Procrustes de landmarks y semilandmarks fueron incluidas como variables dependientes y el logaritmo del Tamaño Centroide (log-TC) como independiente (Drake y Klingenberg, 2008; Mitteroecker y Gunz, 2009). La variación morfométrica en la forma total, así como en el componente no alométrico, fue resumida empleando un Análisis de Componentes Principales (ACP) basado en una matriz 
de covarianza de las coordenadas Procrustes de landmarks y semilandmarks y de los residuales de la Regresión Multivariada, respectivamente (Adams, Rohlf y Slice, 2004; Mitteroecker y Gunz, 2009). Los scores del ACP describen la mayor variación de forma entre los individuos que componen la muestra estudiada. El ACP y la Regresión Multivariada fueron efectuados mediante el programa MorphoJ 1.06d (Klingenberg, 2011).

Finalmente, se estimó la señal filogenética y se mapearon el tamaño, los scores del ACP de forma, así como del componente alométrico y el no-alométrico, sobre un árbol crono-filogenético molecular del clado estimado con secuencias de citocromo $b$ (Cyt $b$ ) y $12 \mathrm{~S}$ del ADN mitocondrial (Material suplementario 1; Lynch Alfaro et al., 2012a). Estos análisis permiten conocer el grado de estructura filogenética en las distintas variables morfométricas estudiadas (Blomberg et al., 2003). El árbol crono-filogenético fue estimado empleando BEAST 1.6.1 (Drummond y Rambaut, 2007), considerando el modelo de substitución TN93+G+I, un modelo lognormal auto-correlacionado para el reloj molecular y una cadena de Markov Monte Carlo de 20 millones de longitud. El cálculo de la señal filogenética y el mapeamiento de las variables sobre el árbol crono-filogenético fue efectuado empleando los paquetes ape, picante y phytools del programa R (R Development Core Team, 2017).

\section{RESULTADOS Y DISCUSIÓN}

Los resultados del Análisis de Componentes Principales sobre las variables de forma calculadas con los datos de landmarks muestran que las especies de Cebus y Sapajus no se diferencian claramente (Fig. 2A). De la misma manera, el ACP sobre las variables forma de landmarks y semilandmarks obtenidas con el criterio de Bending Energy muestran que todas las especies se encuentran superpuestas a lo largo del CP 1, que explica el 26,01\% de la variación (Fig. 2B). A su vez se observa que hay diferenciación entre las especies de ambos géneros a lo largo del CP $2(23,80 \%$ de la variación total en forma; Fig. 2B). Este resultado sugiere que los datos de forma basados en landmarks y semilandmarks, que describen los contornos de la mandíbula, presentan mayor cantidad de información acerca de las diferencias morfométricas entre los géneros. Es notable en la Figura 2B que, cuando consideramos ambos CPs de manera conjunta, existe diferenciación tanto entre especies del mismo género (principalmente para Cebus) como entre especies de diferentes géneros. Por otro lado, se puede observar una marcada similitud en forma entre las especies $S$. apella y $S$. macrocephalus por un lado y $S$. libidinosus y $S$. robustus por el otro. Las especies de $S$. nigritus y $S$. cay presentan una distribución aproximadamente intermedia de morfologías entre los grupos de Sapajus descritos anteriormente (Fig. 2B). Debido a que los datos de landmarks y semilandmarks mostraron un mayor poder para describir las diferencias entre los géneros, los análisis subsiguientes se efectúan sobre este conjunto de datos.

Los cambios de forma observados con el análisis de componentes principales se muestran en la Figura 3 y el Material suplementario 2. Las mandíbulas del extremo positivo del CP1 -correspondientes con individuos de ambos géneros, pero con preponderancia de Sapajusposeen un cuerpo mandibular más alto y relativamente más corto, una rama mandibular más ancha y más alta, un gonion más desarrollado, un cóndilo mandibular de tamaño relativamente menor y una apófisis coronoides más grande que los valores negativos del mismo CP (Fig. 3). A lo largo del CP 2, en el cual se diferencian los dos géneros, las diferencias morfométricas en la mandíbula no son tan marcadas como en el CP1. Las mandíbulas en el extremo negativo del CP2 presentan un cuerpo mandibular relativamente más alto, una rama mandibular más alta, la región del gonion está menos desarrollada, el cóndilo mandibular es más prominente y la apófisis coronoides es relativamente más larga y delgada en comparación con el extremo positivo del mismo CP (Fig. 3).

Es importante remarcar que las diferencias observadas en el CP2 de las coordenadas de forma Procrustes, que han permitido diferenciar los géneros en nuestro análisis, no son las descriptas por otros investigadores como las características de esta diferenciación. En este sentido, el análisis de Regresión Multivariada (Fig. 4A) mostró que el tamaño sólo explica alrededor de un $19 \%$ de la variación en forma, pero que al excluir el componente alométrico en el ACP, la variación residual o no-alométrica muestra una 

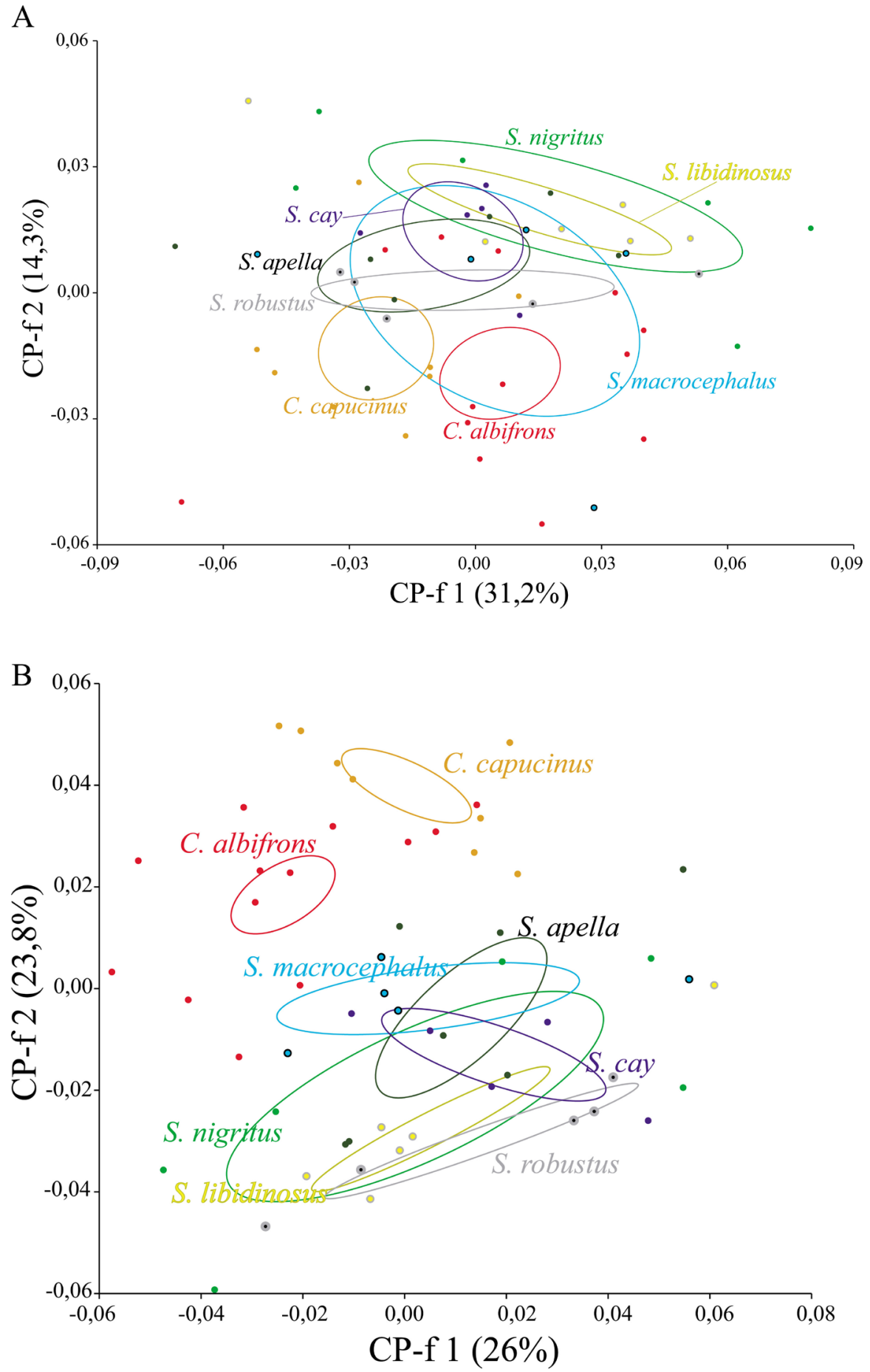

Fig. 2. Gráficos de dispersión de los Componentes Principales 1 y 2 calculados con las coordenadas de forma Procrustes (CP-f1 y CP-f2, respectivamente) de los datos de landmarks (A) y landmarks y semilandmarks (B), mostrando las diferencias entre individuos asignados a ambos géneros. Los casos están agrupados por especie y las elipses representan el intervalo de confianza del $70 \%$ del consenso de cada especie. 

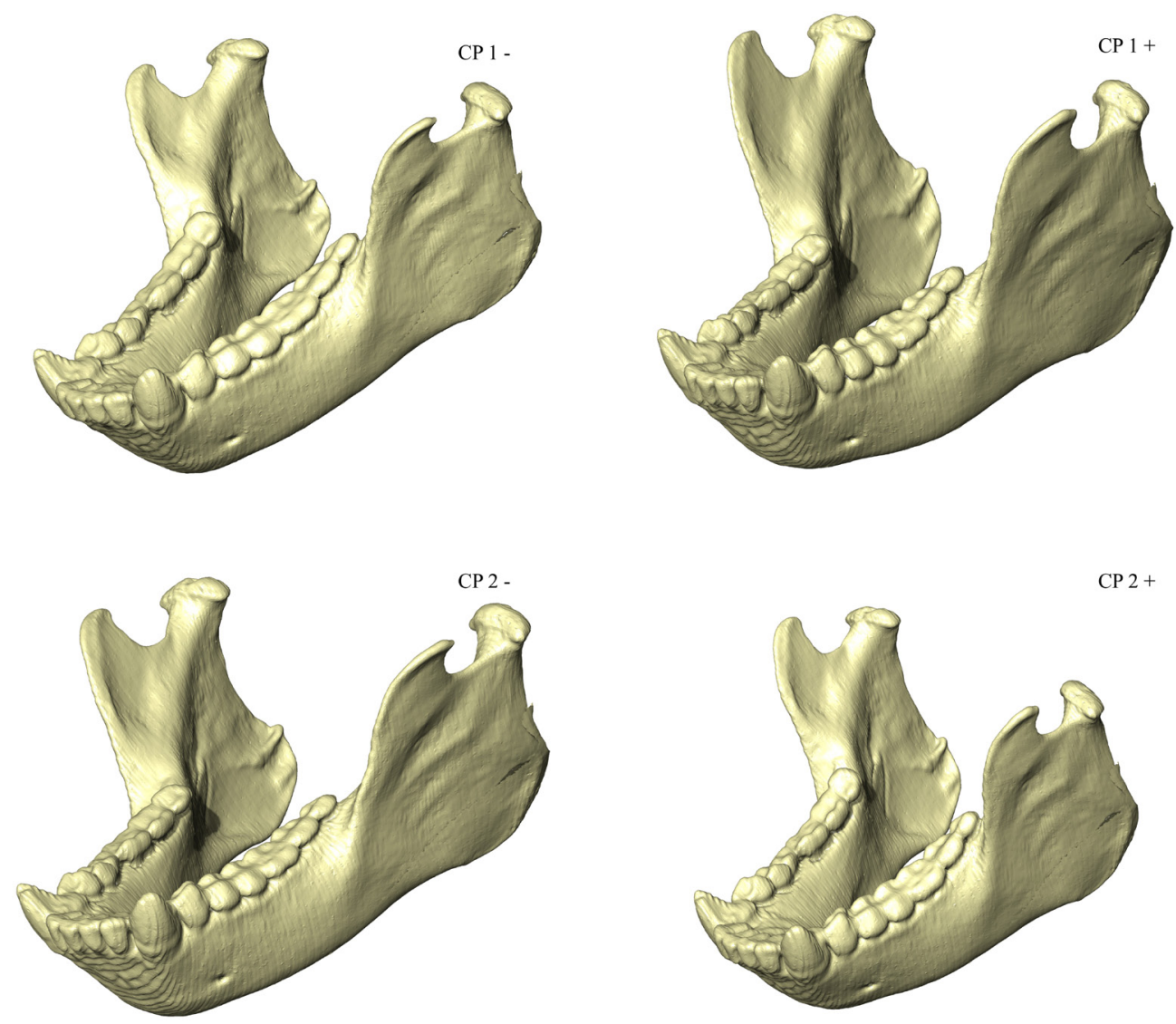

CP $2+$

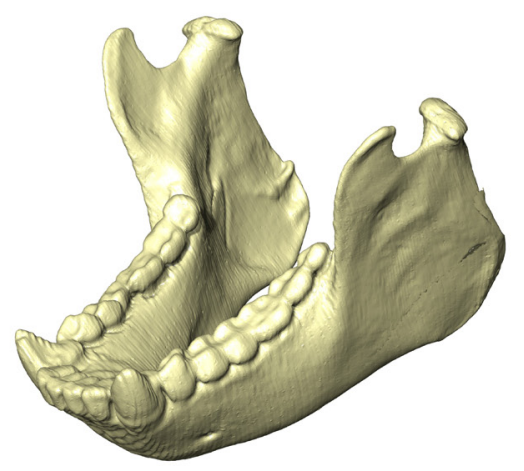

Fig. 3. Cambios morfométricos en la mandíbula observados a lo largo del CP1 y del CP2.

clara diferenciación de las especies de Cebus de las de Sapajus (Fig. 4B). Cuando mapeamos todas estas características morfométricas sobre el árbol filogenético del clado (Fig. 5) podemos observar que el CP 2 del análisis de forma y el CP 1 de componente no-alométrico de variación en forma muestran el mayor ajuste sobre la filogenia. Este patrón también es sustentado por el análisis de señal filogenética (Tabla 3), que muestra valores significativos y cercanos a la expectativa por Movimiento Browniano para el CP2 del análisis de forma y el CP1 de componente no-alométrico.

El patrón de distribución en el espacio de forma de las especies y los especímenes asignados a ambos géneros, hace difícil caracterizar únicamente con base en la morfología de las mandíbulas a ambos géneros como grupos marcadamente diferenciados, contradiciendo hipótesis planteadas en trabajos previos (Lynch
Alfaro et al., 2012b; Wright et al., 2015). Estos trabajos plantean que los especímenes de género Cebus pueden ser agrupados en dos clados (dos géneros para muchos autores) caracterizados por diferencias morfológicas marcadas en su robustez del cráneo y, principalmente, de la mandíbula (Lynch Alfaro et al., 2012b; Wright et al., 2015). Las características morfométricas descritas por el CP1 y en el score de cambio alométrico calculado en este trabajo, que en gran medida se relacionan con cambios en la robustez, no pueden ser empleadas para diferenciar claramente ambos géneros. En cambio, la variación en forma mandibular observada entre los géneros se relacionada con características que son independiente de los cambios en el tamaño de los individuos y que son probablemente explicables por el tiempo de divergencia $\mathrm{y}$ aislamiento reproductivo entre las especies estudiadas (Fig. 5; Tabla 3). Estos resultados 

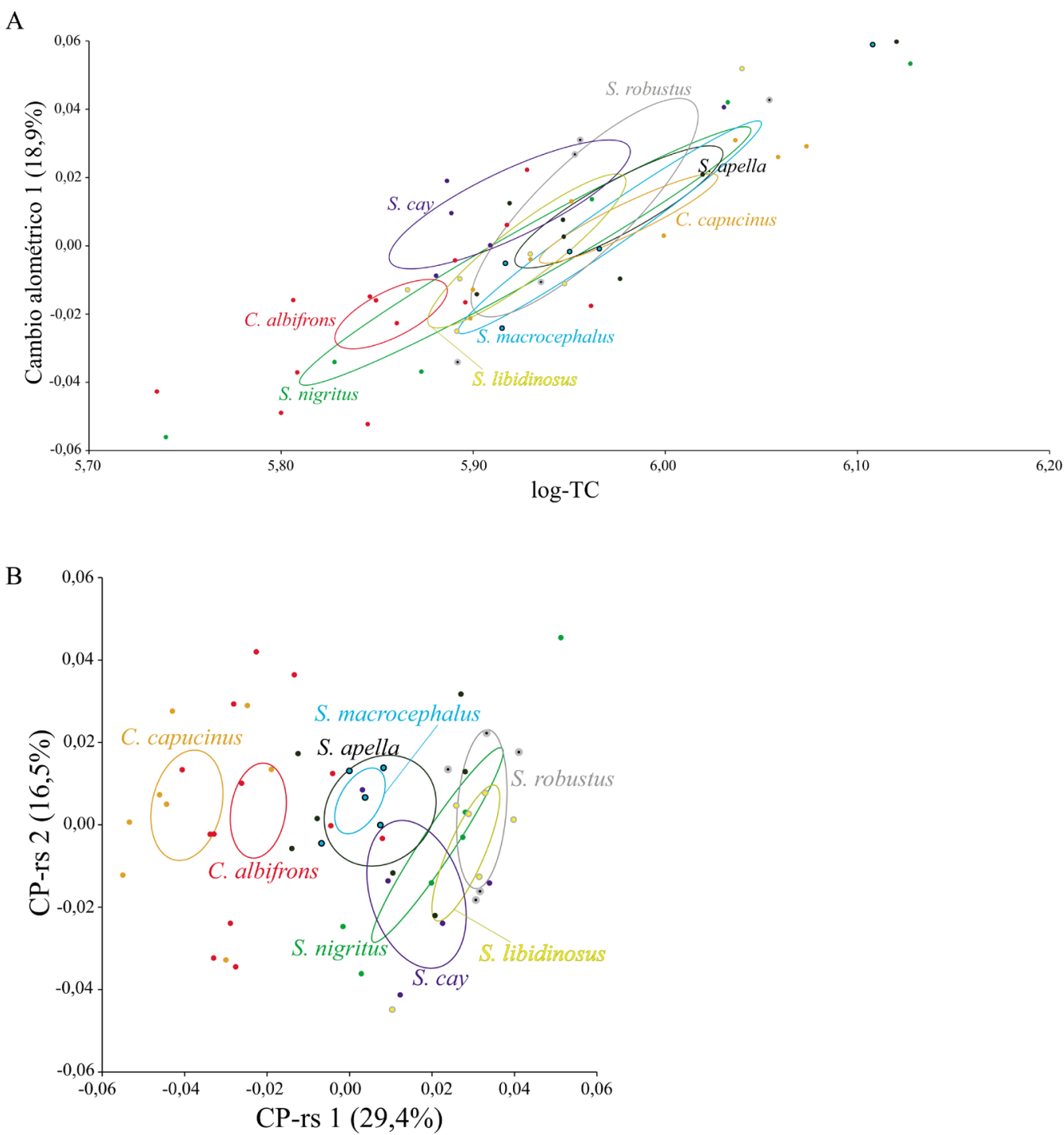

Fig. 4. Gráficos de dispersión del logaritmo del Tamaño Centroide (logTC) versus el cambio alométrico predicho por el modelo de Regresión Multivariada (A) y de los Componentes Principales 1 y 2 calculados con los residuales (CP-rs 1 y CP-rs 2, respectivamente) de los landmarks y semilandmarks (B), mostrando las diferencias entre individuos asignados a ambos géneros. Los casos están agrupados por especie y las elipses representan el intervalo de confianza del $70 \%$ del consenso de cada especie.

concuerdan con los obtenidos con técnicas de morfometría geométrica sobre los molares 1 y 2 de las mismas especies estudiadas en este trabajo y que han sido interpretados principalmente como cambios graduales a lo largo del proceso de divergencia filogenética, entre otros factores (Nova Delgado et al., 2015).

Por otro lado, en nuestros resultados se puede observar que las especies de Cebus presentan una gran distribución en el CP1, extendiéndose principalmente en el extremo negativo, pero también en el positivo, y esto se observa también en los especímenes asignados a Sapajus (Fig. 2). Este resultado sugiere que el grupo de especies analizado es extremadamente variado en cuanto a su fenotipo, tanto a nivel intra como inter-género. Este resultado está en concordancia con un estudio molecular reciente que muestra que no 

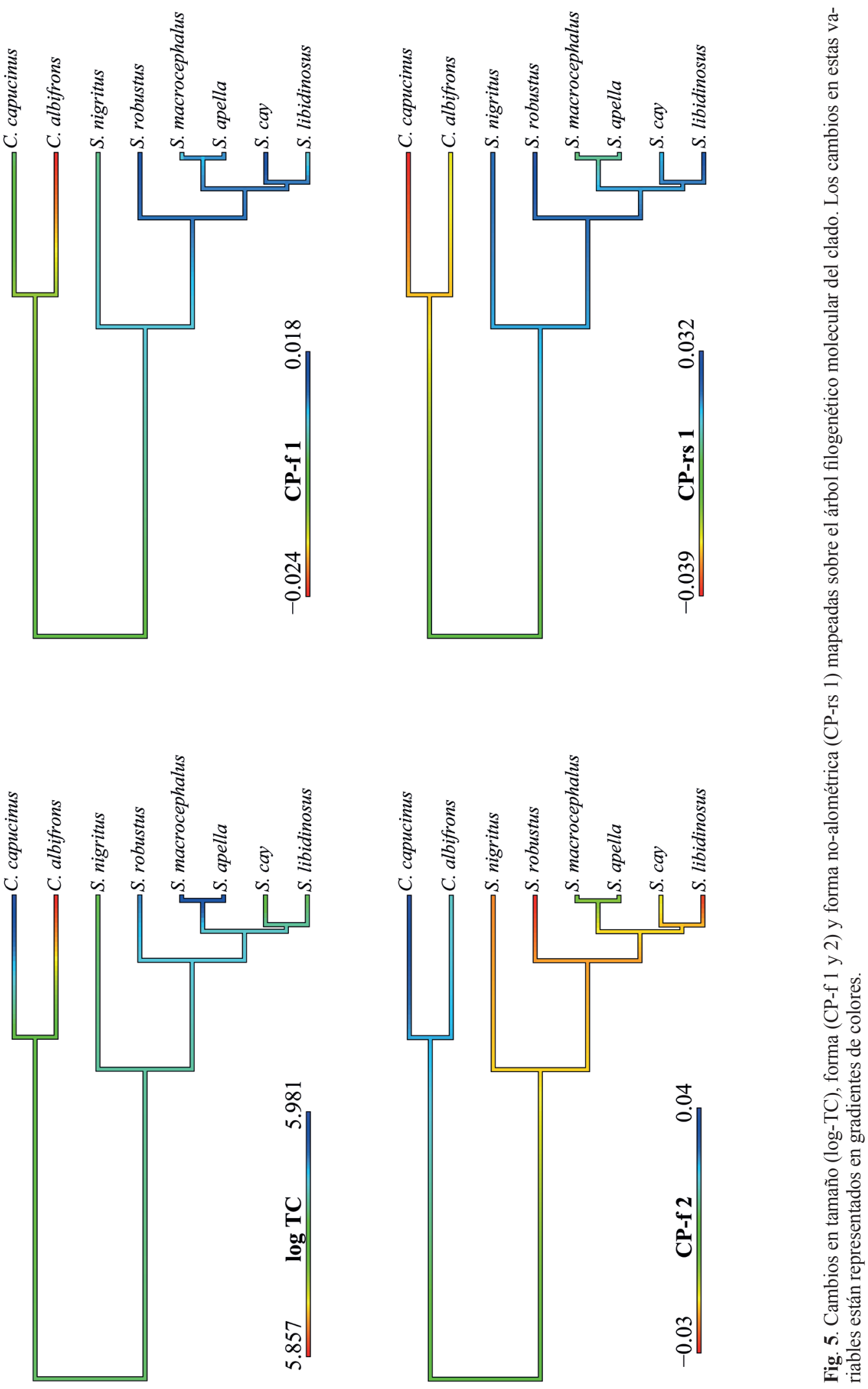
TABLA 3. Resultados del análisis de señal filogenética $(K)$

\begin{tabular}{ccc}
\hline Variable* $^{*}$ & $\mathrm{~K}$ & $\mathrm{p}$ \\
\hline $\operatorname{logTC}$ & 0.355 & 0.080 \\
CP-f 1 & 0.362 & 0.153 \\
CP-f 2 & $\mathbf{0 . 7 8 7}$ & $\mathbf{0 . 0 0 4}$ \\
CP-rs 1 & $\mathbf{0 . 8 4 2}$ & $\mathbf{0 . 0 0 4}$ \\
\hline
\end{tabular}

*log-TC:logaritmo del Tamaño Centroide; CP-f 1 y CP-f 2:Componentes Principales 1 y 2 de las coordenadas de forma Procrustes); CP-rs 1:Componente Principal 1 de los residuales del modelo de Regresión Multivariada. Valores significativos están indicados en negrita.

se encuentra congruencia entre la variación en el ADN mitocondrial y la diferenciación de muchas de las especies de Sapajus (i.e., S. flavius, $S$. cay, S. macrocephalus, S. libidinosus y $S$. apella) definidas con criterios morfológicos, sugiriendo que las mismas forman juntas un único clado con sub-estructuración geográfica y una antigüedad Pleistocénica (Lima et al., 2017).

Resumiendo, los cambios en forma observados en este trabajo estudiando imágenes tridimensionales y empleando técnicas de morfometría geométrica no se correlacionan con lo descripto por otros investigadores empleando morfometria tradicional y técnicas morfoscópicas (Lynch Alfaro et al., 2012b; Silva, 2001; Wright et al., 2015). En estos trabajos caracterizan a las mandíbulas de Sapajus por poseer un cuerpo mandibular más robusto y corto, una rama mandibular de mayor tamaño con un gonion pronunciado y una base mandibular más curva que la observada en las especies asignadas al género Cebus. Estas características no se observan claramente como discriminadoras de los géneros en nuestro trabajo. Sin embargo, muchas de estas características asignadas al recientemente propuesto género Sapajus, que señalan particularmente la robustez de la mandíbula, son observadas en los machos de especies que han sido asignadas a ambos géneros. Sólo la variación no-alométrica muestra una fuerte estructura filogenética en el clado, diferenciando claramente las especies de ambos géneros. Estos resultados sugieren que las características morfológicas descritas previamente para diferenciar los géneros de capuchinos deben ser empleadas con cuidado como criterios válidos para generar nuevas propuestas sistemáticas. Asimismo, sugieren la necesidad de llevar adelante futuros estudios para conocer la influencia de factores aleatorios y determinísticos (e.g., ecológicos) en la diversificación en la morfología mandibular, así como de otras estructuras anatómicas, de las especies de monos capuchinos y de esta manera discutir con un sustento más sólido la sistemática evolutiva del clado.

\section{AGRADECIMIENTOS}

Los autores agradecen a dos revisores anónimos y a la Editora Asociada por los comentarios sobre versiones previas de este trabajo que ayudaron a mejorarlo de manera significativa.

\section{LITERATURA CITADA}

Adams, D. C. y Otarola-Castillo, E. (2013). geomorph: an R package for the collection and analysis of geometric morphometric shape data. $\mathrm{Me}$ thods in Ecology and Evolution, 4, 393-399. doi: 10.1111/2041-210X.12035

Adams, D. C., Rohlf, F. J. y Slice, D. E. (2004). Geometrics morphometrics: ten years of progress following the 'revolution'. Italian Journal of Zoology, 71, 5-16. doi: 10.1080/11250000409356545

Arístide, L., Soto, I. M., Mudry, M. D. y Nieves, M. (2014). Intra and interspecific variation in cranial morphology on the southernmost distributed Cebus (Platyrrhini, Primates) species. Journal of Mammalian Evolution, 21, 349-355. doi: 10.1007/s10914-013-9249-y

Aristide, L., dos Reis, S. F., Machado, A.C., Lima, I., Lopes, R. T. y Perez, S. I. (2015). Encephalization and diversification of the cranial base in platyrrhine primates Journal of Human Evolution, 81, 29-40. doi: 10.1016/j. jhevol.2015.02.003

Aristide, L., dos Reis, S. F., Machado, A. C., Lima, I., Lopes. R. T. y Perez, S. I. (2016). Brain shape convergence in the adaptive radiation of New World Monkeys. Proceedings of the National Academy of Sciences USA, 113, 2158-2163. doi: 10.1073/pnas. 1514473113

Blomberg, S. P., Garland Jr., T. e Ives, A.R. (2003). Testing for phylogenetic signal in comparative data: behavioral traits are more labile. Evolution, 57, 717-745.

Drake, A. G. y Klingenberg, C. P. (2008). The pace of morphological change: historical transformation of skull shape in St Bernard dogs. Proceedings of the Royal Society B: Biological Sciences, 275, 71-76. doi: 10.1098/ rspb.2007.1169

Drummond, A. J. y Rambaut, A. (2007). BEAST: Bayesian evolutionary analysis by sampling trees. BMC Evolutionary Biology, 7, 214. doi: 10.1186/1471-2148-7-214

Felsenstein, J. (1985). Phylogenies and the comparative method. American Naturalist, 125, 1-15.

Fleagle, J. G. (2013). Primate adaptation and evolution. San Diego, USA: Academic Press. 
Klingenberg, C. P. (2011). MorphoJ: an integrated software package for geometric morphometrics. Molecular Ecology Resources, 11, 353-357. doi: 10.1111/j.17550998.2010.02924.x

Lima, M. G., Buckner, J. C., Aleixo, A., Martins, A. B., Boubli, J. P., Link, A., Farias, I. P., da Silva, M. N., Röhe, F., Queiroz, H., Chiou, K.L., Di Fiore, A., Alfaro, M. E. y Lynch Alfaro, J. W. (2017). Capuchin monkey biogeography: understanding Sapajus Pleistocene range expansion and the current sympatry between Cebus and Sapajus. Journal of Biogeography, 44, 810-820. doi: 10.1111/jbi.12945

Losos, J. B. y Mahler, D. L. (2010). Adaptive radiation: the interaction of ecological opportunity, adaptation, and speciation. En: Bell, M.A., Futuyma, D. J., Eanes, W.F. y Levinton, J.S., (Eds.). Evolution since Darwin: the first 150 years. (pp. 381-420). Sunderland, United Kingdom: Sinauer Associates.

Lynch Alfaro, J. W., Boubli, J. P., Olson, L. E., Di Fiore, A., Wilson, B., Gutierrez-Espeleta, G. A., Chiou, K. L., Schulte, M., Neitzel, S., Ross, V., Schwochow, D., Farias, I., Janson, C. y Alfaro, M. E. (2012a). Explosive Pleistocene range expansion leads to widespread Amazonian sympatry between robust and gracile capuchin monkeys. Journal of biogeography, 39, 272-288. doi: 10.1111/j.1365-2699.2011.02609.x

Lynch Alfaro, J. W., Silva Jr., J. S y Rylands, A. B. (2012b). How different are robust and gracile capuchin monkeys? An argument for the use of Sapajus and Cebus. American Journal of Primatology, 74, 273-286. doi: 10.1002/ajp.22007

Marroig, G. y Cheverud, J. M. (2001). A comparison of phenotypic variation and covariation patterns and the role of phylogeny, ecology and ontogeny during cranial evolution of New World monkeys. Evolution, 55, 25762600. doi: 10.1554/0014-3820(2001)055[2576:ACOPV A]2.0.CO;2

Mitteroecker, P. y Gunz, P. (2009). Advances in geometric morphometrics. Evolutionary Biology, 36, 235-247. doi: 10.1007/s11692-009-9055-x
Nova Delgado, M., Galbany, J., Górka, K. y Pérez-Pérez, A. (2015). Taxonomic implications of molar morphology variability in Capuchins. International Journal of Primatology, 36, 707-727. doi: 10.1007/s10764-015-9850-4

R Development Core Team. (2017). R: a language and environment for statistical computing. Vienna, Austria: R Foundation for Statistical Computing.

Rohlf, F. J. (1990). Rotational fit (Procrustes) methods. En: Rohlf, F. J. y Bookstein, F. L., (Eds.). Proceedings Michigan morphometrics workshop. Special publication n8 2. Museum of Zoology. (pp. 227-236). Michigan, USA: University of Michigan.

Rosenberger, A. L. (1992). Evolution of feeding niches in New World monkeys. American Journal of Physical Anthropology, 88, 525-562. doi: 10.1002/ajpa.1330880408

Rylands, A. B., Mittermeier, R. A. y Silva, J. S. (2012). Neotropical primates: taxonomy and recently described species and subspecies. International Zoo Yearbook, 46, 11-24. doi: 10.1111/j.1748-1090.2011.00152.x

Schluter, D. (2000). The ecology of adaptive radiation. Oxford, United Kingdom: Oxford University Press.

Silva, J. S, Jr. (2001). Especiacao nos macacos-prego e caiararas, genero Cebus Erxleben, 1777 (Primates, Cebidae). (Tesis doctoral). Universidade Federal do Rio de Janeiro. Rio de Janeiro.

Wiley, D. F., Amenta, N., Alcantara, D. A., Ghosh, D., Kil, Y. J., Delson, E., Harcourt-Smith, W., Rohlf, F. J., St. John, K., Hamann, B., Motani, R., Frost, S., Rosenberger, A. L., Tallman, L., Disotell, T. y O’Neill, R. (2005). Evolutionary morphing. En: Proceedings of the IEEE Visualization 2005 (VIS '05). Minneapolis, USA. p. 431-438.

Wright, K. A., Wright, B. W., Ford, S.M., Fragaszy, D., Izar, P., Norconk, M., Masterson, T., Hobbs, D. G., Alfaro, M. E. y Alfaro, J. W. L. (2015). The effects of ecology and evolutionary history on robust capuchin morphological diversity. Molecular Phylogenetics and Evolution, 82, 455-466. doi: 10.1016/j.ympev.2014.08.009 